\title{
Article \\ A Generalized Bochner Technique and Its Application to the Study of Conformal Mappings
}

\author{
Vladimir Rovenski $^{1, *(\mathbb{D}}$, Sergey Stepanov ${ }^{2}$ and Irina Tsyganok $^{2}(\mathbb{D}$ \\ 1 Department of Mathematics, University of Haifa, Mount Carmel, Haifa 3498838, Israel \\ 2 Department of Mathematics, Finance University, 49-55, Leningradsky Prospect, 125468 Moscow, Russia; \\ s.e.stepanov@mail.ru (S.S.); i.i.tsyganok@mail.ru (I.T.) \\ * Correspondence: vrovenski@univ.haifa.ac.il
}

check for

updates

Citation: Rovenski, V.; Stepanov, S.; Tsyganok, I. A Generalized Bochner Technique and Its Application to the Study of Conformal Mappings. Axioms 2021, 10, 333. https:// doi.org/10.3390/axioms10040333

Academic Editor: Anna Maria Fino

Received: 4 November 2021

Accepted: 3 December 2021

Published: 5 December 2021

Publisher's Note: MDPI stays neutral with regard to jurisdictional claims in published maps and institutional affiliations.

Copyright: (C) 2021 by the authors Licensee MDPI, Basel, Switzerland. This article is an open access article distributed under the terms and conditions of the Creative Commons Attribution (CC BY) license (https:// creativecommons.org/licenses/by/ $4.0 /)$.

\begin{abstract}
This article is devoted to geometrical aspects of conformal mappings of complete Riemannian and Kählerian manifolds and uses the Bochner technique, one of the oldest and most important techniques in modern differential geometry. A feature of this article is that the results presented here are easily obtained using a generalized version of the Bochner technique due to theorems on the connection between the geometry of a complete Riemannian manifold and the global behavior of its subharmonic, superharmonic, and convex functions.
\end{abstract}

Keywords: Bochner technique; Riemannian and Kähler manifolds; conformal diffeomorphism; Liouville-type theorem; scalar curvature; paracomplex structure

MSC: $53 C 20$

\section{Introduction}

The prototype of the generalized Bochner technique is the celebrated classical Bochner technique, first introduced by S. Bochner, K. Yano, A. Lichnerowicz, and others in the 1950s and 1960s to study the relationship between the topology and curvature of a compact boundaryless Riemannian manifold (see [1]). This method is used to prove the vanishing theorem for the kernel of the Laplace operator admitting a Weitzenböck decomposition on compact manifolds (see [2] (p. 53)). As a result, we have a number of theorems based on the classical Bochner technique, which usually show that the assumption of positive or negative curvature sectional curvatures of compact Riemannian manifolds yields the vanishing of some geometrically interesting tensor fields and mappings. The most famous results of the classical Bochner technique are the theorem of D. Meyer and S. Gallot (see [3]) on the vanishing of Betti numbers of compact Riemannian manifolds and the theorem of J. Eells and J. H. Sampson (see [4] (p. 465)) on the absence of harmonic mappings of compact Riemannian manifolds.

The classical Bochner technique is used in a number of articles, monograph chapters, and analytical reviews (see, for example, [1]; [4]; [5] (pp. 333-363); [6,7]). On the other hand, since the 1970s, complete (non-compact) Riemannian manifolds have been included in the circle of research carried out using the Bochner technique. For this, methods of geometric analysis have been developed (see, for example, [4] (pp. 361-394); [8]; [9,10]). As a result, vanishing theorems for the classical Bochner technique took the form of Liouville-type theorems. The first outstanding achievement in this direction was the theorem of S.-T. Yau and R. Schoen (see [11]), which generalized the result of J. Eells and J. H. Sampson to the case of complete Riemannian manifolds. The new research method developed by S.-T. Yau, R. Schoen, H. Wu, P. Li, and others was later called the generalized Bochner technique (see, for example, [8]). This method studies the relationship between the geometry of a complete Riemannian manifold and the behavior of its convex, subharmonic, and superharmonic functions under the assumptions about either the curvature or the growth of the volume 
of geodesic balls. Today, the new research method is not as popular among geometers as the classical Bochner method. Despite the availability of monographs (e.g., [4,8-10]), there are not many practical applications. In this article, we discuss the global geometry of conformal mappings of complete Riemannian and Kähler manifolds using a generalized version of the Bochner technique. This article continues the series of works $[12,13]$ and can also demonstrate to both newcomers to the field and experienced geometers various methods of the generalized Bochner technique for research on the example of conformal mappings. All new results of the article are easily proved on the basis of known results of geometric analysis, e.g., [1,4,5,7-10], so we only give a sketch of their proofs.

The article is organized as follows. In Section 2, along with basic information on conformal mappings, we demonstrate the classical Bochner technique with examples for compact manifolds. In the other four sections, we demonstrate applications of various methods of the generalized Bochner technique to the study of conformal diffeomorphisms of complete Riemannian manifolds.

\section{Preliminaries on Conformal Mappings and the Classical Bochner Technique}

Let $(M, g)$ and $(\bar{M}, \bar{g})$ be two $n$-dimensional $(n \geq 3)$ connected Riemannian manifolds with the Levi-Civita connections $\nabla$ and $\bar{\nabla}$, respectively, and let $f: M \rightarrow \bar{M}$ be a diffeomorphism of $M$ onto $\bar{M}$. Suppose that $x$ is any point of $M$; then, by [14] (Theorem 2.2.11), there exist a neighborhood $U \subset M$ of this point with local coordinates $x^{1}, \ldots, x^{n}$ and a neighborhood $\bar{U} \subset \bar{M}$ of $\bar{x}=f(x) \in \bar{M}$ with local coordinates $\bar{x}^{1}, \ldots, \bar{x}^{n}$ such that the diffeomorphism $\left.f\right|_{U}$ is given by the equalities $\bar{x}^{1}=x^{1}, \ldots, \bar{x}^{n}=x^{n}$. In this case, an arbitrary pair of points $y \in U$ and $\bar{y}=f(y) \in f(U)$ must have the same coordinates. We will use such special local coordinate systems as needed throughout this article (see also $[1,6]$ ).

Recall that a diffeomorphism $f: M \rightarrow \bar{M}$ is a conformal mapping $f:(M, g) \rightarrow(\bar{M}, \bar{g})$ of Riemannian manifolds if there is a smooth function $\sigma$ on $M$ such that $f^{*} \bar{g}=e^{2 \sigma} g$, i.e., the pull back of the metric $\bar{g}$ is proportional to $g$ (see [15]). The last equations can be rewritten equivalently as

$$
\bar{g}_{i j}=e^{2 \sigma} g_{i j},
$$

where $g_{i j}=g\left(\partial / \partial x^{i}, \partial / \partial x^{j}\right)$ and $\bar{g}_{i j}=\bar{g}\left(\partial / \partial x^{i}, \partial / \partial x^{j}\right)$ with respect to common local coordinates $x^{1}, \ldots, x^{n}$ (see also [16] (p. 89)). In other words, we suppose that all objects under consideration (as connections, tensor fields, etc.), with bar or without, are defined on the same underlying manifold. In particular, if $\sigma$ is constant, then $f$ is a homothetic mapping, and if $\sigma=0$, then $f$ is an isometry. If we denote by Ric and $\overline{\text { Ric }}$ the Ricci tensors of $(M, g)$ and $(\bar{M}, \bar{g})$, respectively, then for a conformal mapping $f:(M, g) \rightarrow(\bar{M}, \bar{g})$, the following equations hold (see [15]; [16] (p. 90)):

$$
\bar{R}_{i j}=R_{i j}-(n-2)\left(\nabla_{i}\left(\partial_{j} \sigma\right)-\partial_{i} \sigma \partial_{j} \sigma\right)+\left(\Delta \sigma+(n+2)\|\operatorname{grad} \sigma\|^{2}\right) g_{i j},
$$

where $\bar{R}_{i j}$ and $R_{i j}$ are components of the Ricci tensors $\overline{R i c}$ and Ric, respectively; $\nabla_{i}=\nabla_{\partial / \partial x^{i}}$ and $\|\operatorname{grad} \sigma\|^{2}=g(\operatorname{grad} \sigma, \operatorname{grad} \sigma)$ for $(\operatorname{grad} \sigma)^{i}=\partial^{i} \sigma=g^{i j} \partial_{j} \sigma$ with the components $g^{k l}$ of the inverse of the metric tensor $g$ and $\partial_{i} \sigma=\partial \sigma / \partial x^{i}$. Here, $\Delta \sigma=\operatorname{div}(\operatorname{grad} \sigma)$ is the well-known Laplace-Beltrami operator, similar to Laplacian (see, for example, [5] (p. 61;74, etc.)). From (2), we conclude that if $f:(M, g) \rightarrow(\bar{M}, \bar{g})$ is a homothetic mapping, then $\overline{R i c}=f^{*}$ Ric. If we denote by $\bar{s}$ and $s$ the scalar curvatures of $(M, g)$ and $(\bar{M}, \bar{g})$, respectively, then contracting (2), we obtain the following equation (see [15] and [16] (p. 90)):

$$
e^{2 \sigma} \bar{s}=s-2(n-1) \Delta \sigma-(n-1)(n-2)\|\operatorname{grad} \sigma\|^{2} .
$$

The Schouten tensor $S$ of $(M, g)$ is introduced by the following equality:

$$
S=\frac{1}{n-2}\left(\operatorname{Ric}-\frac{s}{2(n-1)} g\right) .
$$


The Schouten tensor is especially important for conformal geometry because of its relatively simple conformal diffeomorphism law

$$
\bar{S}_{i j}=S_{i j}-\nabla_{i}\left(\partial_{j} \sigma\right)+\partial_{i} \sigma \partial_{j} \sigma-(1 / 2)\|\operatorname{grad} \sigma\|^{2} g_{i j} .
$$

Note that (4) is a direct consequence of (2) and (3). From (4), we conclude that if $f:(M, g) \rightarrow$ $(\bar{M}, \bar{g})$ is a homothetic mapping, then $f^{*} \bar{S}=S$.

Now, let $(M, g)$ be compact. We can assume that $s \leq 0$ and $\bar{s} \geq 0$. Thus, from (3), it follows that $\Delta \sigma \leq 0$. Then, by Bochner's lemma (see [1] (p. 30) and Remark 3), we obtain $\sigma=$ const and $\Delta \sigma=0$, and again by (3) and our assumptions, $s=\bar{s}=0$. Setting

$$
\sigma=\frac{2}{n-2} \ln u
$$

for a smooth scalar function $u>0$, from $\bar{g}=e^{2 \sigma} g$, we obtain

$$
\bar{g}=u^{4 /(n-2)} g, \quad u>0,
$$

where $u$ is called the associated function of the conformal diffeomorphism $f:(M, g) \rightarrow$ $(\bar{M}, \bar{g})$ (see [15]). In this case, (3) can be rewritten in the following form (see [2] (p. 59)):

$$
\Delta u=\frac{n-2}{4(n-1)}\left(u s-u^{\frac{n+2}{n-2}} \bar{s}\right) .
$$

When $M$ is compact, integrating (5) over $(M, g)$ and using the Green theorem (see [1] (p. 31)) gives

$$
\int_{M} s u d \operatorname{vol}_{g}=\int_{M} u^{\frac{n+2}{n-2}} \bar{s} d \operatorname{vol}_{g} .
$$

Assuming $\bar{s} \leq 0$ and $s \geq 0$ for the above integral formula, we obtain $\bar{s}=s=0$. Thus, from (3), we can conclude that $\sigma=$ const. Hence, the conformal diffeomorphism $f:(M, g) \rightarrow$ $(\bar{M}, \bar{g})$ is a homothetic mapping. Therefore, we can formulate the following theorem.

Theorem 1. Let $(M, g)$ be a compact without boundary Riemannian manifold of dimension $n \geq 3$ with scalar curvature $s \leq 0$ (or $s \geq 0$ ), and let $(\bar{M}, \bar{g})$ be another Riemannian manifold with scalar curvature $\bar{s} \geq 0$ (or $\bar{s} \leq 0$, respectively). If there exists a conformal diffeomorphism $f:(M, g) \rightarrow(\bar{M}, \bar{g})$, then it is a homothetic mapping; moreover, $(M, g)$ and $(\bar{M}, \bar{g})$ have zero scalar curvature.

Remark 1. For the two-dimensional case, a conformal diffeomorphism $f$ (in Theorem 1) is just a holomorphic transformation between the underlying complex structures of $M$ and $\bar{M}$. Since the scalar curvature is twice the Gaussian curvature, $\chi(M) \leq 0$ (or $\chi(M) \geq 0$ ) and $\chi(\bar{M}) \geq 0$ (or $\chi(\bar{M}) \leq 0$, respectively) by Gauss-Bonnet theorem; thus, $\chi(M)=\chi(\bar{M})=0$. Therefore, $s=\bar{s}=0$.

Remark 2. Applying the Green theorem (see [1] (p. 31)) to $\Delta \sigma$, we obtain

$$
\int_{M} \Delta \sigma d \operatorname{vol}_{g}=0
$$

where $d \mathrm{vol}_{g}$ is the Riemannian volume form. Then, integrating (3) over $(M, g)$ gives

$$
s(M)=\int_{M} e^{2 \sigma} \bar{s} d \operatorname{vol}_{g}+(n-1)(n-2) \int_{M}\|\operatorname{grad} \sigma\|^{2} d \operatorname{vol}_{g},
$$

where $s(M)=\int_{M} s d \mathrm{vol}_{g}$ is the total scalar curvature of $(M, g)$ (see [2] (p. 119)). An analysis of the above formulas allows us to conclude that the condition $s \leq 0$ in Theorem 1 can be replaced by the weaker condition $s(M) \leq 0$ (see [15] (Theorem 2)). 
The following corollary of Theorem 1 generalizes Yau's similar statement [15] (Corollary 2.1) with constant scalar curvature.

Corollary 1. Let $g$ and $\bar{g}$ be two conformally equivalent metrics on an $n$-dimensional $(n \geq 3)$ compact manifold $M$. If both metrics have nonvanishing scalar curvatures, i.e., $s \neq 0$ and $\bar{s} \neq 0$ everywhere, then these scalar curvatures have the same sign.

\section{An Application of the Hopf Maximum Principle to the Study of Conformal Mappings}

There are various formulations of the maximum principle, from its classical Hopf form up to generalizations of the Omori-Yau maximum principle at infinity in [9], where applications are given to a number of problems in the context of complete Riemannian manifolds, under the assumption of either curvature or the volume growth of geodesic balls. This is a part of the generalized Bochner technique. The Hopf maximum principle in the theory of second order elliptic differential equations on Riemannian manifolds (e.g., [2]) tells us that "if $\Delta \phi \geq 0$ and $\phi$ attains a local maximum value at some point, then $\phi$ is constant", and it has been described as the "classical and bedrock result" of that theory. Here, we consider an application of the maximum principle of E. Hopf to the classical theory of conformal mappings.

Theorem 2. Let $(M, g)$ be a Riemannian manifold of dimension $n \geq 3$ with scalar curvature $s \geq 0$, and let $(\bar{M}, \bar{g})$ be another Riemannian manifold with scalar curvature $\bar{s} \leq 0$. Suppose that there exists a conformal diffeomorphism $f:(U, g) \rightarrow(\bar{U}, \bar{g})$ for connected domains $U \subset M$ and $\bar{U} \subset \bar{M}$ such that $\bar{g}=e^{2 \sigma} g$ on $\mathcal{U}$. If the function $-\sigma$ attains a local maximum at some point $x \in U$, then $f$ is a homothetic mapping; moreover, $(M, g)$ and $(\bar{M}, \bar{g})$ have zero scalar curvature on $U$ and $\bar{U}$, respectively.

Proof. The function $\phi=-\sigma$ on $U$ satisfies the following differential equation (see (3)):

$$
\Delta \phi=\frac{1}{2(n-1)}\left(e^{-2 \phi} \bar{s}-s\right)+\frac{1}{2}(n-2) g(\nabla \phi, \nabla \phi) .
$$

Assuming $s \leq 0$ and $\bar{s} \geq 0$ for the scalar curvatures on $U$ and $\bar{U}=f(U)$, respectively, we get $\Delta \phi \geq 0$ for $n \geq 3$. Hence, if $\phi$ attains a local maximum at some point of $U$, then by the Hopf maximum principle, $\phi$ is constant on $U$. Then, from (6), we conclude that $\sigma$ is constant on $U$. Hence, $f$ is a homothetic mapping, and $s=\bar{s}=0$ on $U$.

Remark 3. In the conditions of Theorem 2, let $U=M$, where $M$ is a compact manifold without boundary. Then, there exists a point $x \in M$ at which the function $\phi=-\sigma$ reaches its maximum. Thus, the case $s \geq 0$ of Theorem 1 can be regarded as a consequence of Theorem 2.

\section{An Application of the Theory of Superharmonic Functions to the Study of Conformal Mappings}

This section begins with a brief survey of the theory of parabolic manifolds, which is related to superharmonic functions and is part of the generalized Bochner technique. The concept of a parabolic manifold is related to a wide class of equivalent properties of a Riemannian manifold, including Green's kernel, linear capacity, Brownian motion, etc. Thus, there are few equivalent definitions of the parabolicity of a complete Riemannian manifold in various terms (see, for example, [17] (pp. 164-165)). Here is one of the points of view on this concept. Recall that $u \in C^{2}(M)$ is a superharmonic function if $\Delta u \leq 0$. We will say that a Riemannian manifold is a parabolic manifold if it does not admit nonconstant positive superharmonic functions (see, e.g., [10] (p. 313) and [17] (p. 164)) A complete Riemannian manifold of finite volume is an example of a parabolic manifold (see [18]). Let us formulate an analogue of Theorem 1 (see also Remark 2) for the case of complete manifolds. 
Theorem 3. Let $(M, g)$ be a parabolic Riemannian manifold of dimension $n \geq 3$ (in particular, a complete Riemannian manifold of finite volume) with scalar curvature $s \leq 0$, and let $(\bar{M}, \bar{g})$ be another Riemannian manifold with scalar curvature $\bar{s} \geq 0$. If there exists a conformal diffeomorphism $f:(M, g) \rightarrow(\bar{M}, \bar{g})$, then it is a homothetic mapping; moreover, $(M, g)$ and $(\bar{M}, \bar{g})$ have zero scalar curvature.

Proof. From (5), we conclude that if $s \leq 0$ and $\bar{s} \geq 0$, then $\Delta u \leq 0$. Thus, the associated function $u$ of the conformal diffeomorphism $f$ is superharmonic. In addition, if $(M, g)$ is a parabolic Riemannian manifold of dimension $n \geq 3$ (in particular, a complete Riemannian manifold of finite volume), then $u=$ const. In this case, our diffeomorphism $f:(M, g) \rightarrow$ $(\bar{M}, \bar{g})$ is a homothetic mapping.

As another example, we consider conformal diffeomorphisms of complete Kählerian manifolds. First, recall the necessary definitions. Let $(M, J)$ be an almost complex manifold, where $M$ is a connected smooth $2 m$-dimensional manifold (without boundary), and $J$ is a smooth endomorphism of the tangent bundle TM such that $J^{2}=-\mathrm{id}{ }_{T M}$. A Riemannian metric $g$ on $(M, J)$ is Kähler if $g(J, J)=g$ and $\nabla J=0$ for the Levi-Civita connection $\nabla$ of the metric $g$. The triplet $(M, g, J)$ is called a Kähler manifold. Such $(M, g, J)$ has a quasi-positive Ricci curvature if the Ricci curvature is non-negative and is positive at one point of $(M, g, J)$. In turn, $(M, g, J)$ has quasi-negative Ricci curvature if the Ricci curvature is non-positive and is negative at one point of $(M, g, J)$.

Theorem 4 (see [19]). Let $(M, g, J)$ be a complete Kähler manifold with quasi-positive (respectively, quasi-negative) Ricci curvature and the total scalar curvature $s(M)<\infty$ (respectively, $s(M)>$ $-\infty)$; then, $(M, g, J)$ is a parabolic manifold.

Using the above, we can formulate the following corollary.

Corollary 2. Suppose $(M, g, J)$ is a complete Kähler manifold with quasi-negative Ricci curvature and scalar curvature such that $s \leq 0$, and $s(M)>-\infty$. Let $(\bar{M}, \bar{g}, \bar{J})$ be another Kähler manifold with scalar curvature $\bar{s} \geq 0$. If there exists a conformal diffeomorphism $f:(M, g, J) \rightarrow(\bar{M}, \bar{g}, \bar{J})$, then it is a homothetic mapping; moreover, $(M, g, J)$ and $(\bar{M}, \bar{g}, \bar{J})$ have zero scalar curvature.

Note that a parabolic manifold is stochastically complete. Recall that a diffusion process on a Riemannian manifold is conservative or stochastically complete if the associated stochastic process remains forever in the state space. Both stochastic completeness and parabolicity have been the subject of systematic study, e.g., the survey by Grigor'yan [17]. In particular, any complete Riemannian manifold with the Ricci curvature bounded from below by a constant (possibly negative) is stochastically complete (see [20]). Moreover, if $(M, g)$ is stochastically complete, then any non-negative superharmonic function $u \in L^{1}(M, g)$ is constant (see [17] (p. 204)). Therefore, the following theorem is valid.

Theorem 5. Let $(M, g)$ be a complete Riemannian manifold with Ricci curvature bounded from below and scalar curvature $s \leq 0$, and let $(\bar{M}, \bar{g})$ be another Riemannian manifold with scalar curvature $\bar{s} \geq 0$. If there exists a conformal diffeomorphism $f:(M, g) \rightarrow(\bar{M}, \bar{g})$ defined by $f^{*} \bar{g}=e^{2 \sigma} g$, where $\sigma \geq 0$ is a smooth function such that $\sigma \in L^{1}(M, g)$, then $f$ is a homothetic mapping; moreover, $(\bar{M}, g)$ and $(\bar{M}, \bar{g})$ have zero scalar curvature.

Remark 4. In [15] the following was proved: let $(M, g)$ be complete with sectional curvature bounded from below and $s>0$; then, there is no non-homothetic conformal mapping of $(M, g)$ onto a manifold $(\bar{M}, \bar{g})$ with scalar curvature bounded from above by a negative constant. Therefore, Theorem 5 complements this statement and Theorem 1. In addition, we note that the results stated and proved above are new, since no one has considered conformal mappings of parabolic manifolds before us. 


\section{An Application of the Theory of Subharmonic Functions to the Study of Conformal Mappings}

Let $f$ be a conformal diffeomorphism of a complete $n$-dimensional $(n \geq 3)$ Riemannian manifold $(M, g)$ onto another Riemannian manifold $(\bar{M}, \bar{g})$. In particular, if the scalar curvatures of $(M, g)$ and $(\bar{M}, \bar{g})$ satisfy the inequalities $s \geq 0$ and $\bar{s} \leq 0$, respectively, then from (5), we obtain $\Delta u \geq 0$ (see Theorem 1). Thus, the associated function $u$ of the conformal diffeomorphism $f$ is a subharmonic function, since by definition, the function $u \in C^{2}(M)$ is subharmonic if $\Delta u \geq 0$.

Many results on subharmonic functions on complete Riemannian manifolds have been obtained by R. Green and H. Wu, A. Huber, L. Karp, S.-T. Yau, etc. Recall the following famous Liouville-type theorem for subharmonic functions on complete (non-compact) Riemannian manifolds: let $u \geq 0$ be a smooth subharmonic function on $(M, g)$; then,

$$
\int_{M} u^{p} d \operatorname{vol}_{g}=\infty
$$

for any $1<p<\infty$, unless $u$ is a constant (see [21]). In other words, if $u \in L^{p}(M, g)$ for any $1<p<\infty$, then $u$ is a constant $C>0$; hence, $C^{p} \int_{M} d \operatorname{vol}_{g}<\infty$. Therefore, if $\operatorname{Vol}(M, g)=\infty$, then $u \equiv 0$. On the other hand, if $u>0$, then $(M, g)$ has finite volume. Recall that any complete non-compact Riemannian manifold with non-negative Ricci curvature has infinite volume (e.g., [22]). Thus, there are no positive subharmonic $L^{p}(M, g)$ functions for $0<p<\infty$ on a complete non-compact Riemannian manifold with nonnegative Ricci curvature. Using the above, we can generalize Theorem 1 for complete Riemannian manifolds using the theory of subharmonic functions.

Proposition 1. Let $(M, g)$ be a complete Riemannian manifold of infinite volume. Then, it has no positive subharmonic $L^{p}(M, g)$-functions for any $1<p<\infty$. In particular, a complete noncompact Riemannian manifold of non-negative Ricci curvature does not admit positive subharmonic $L^{p}(M, g)$-functions for any $0<p<\infty$.

Proposition 1 is a refinement of the Yau result in [18]. On the other hand, by (5), if $s \geq 0$ and $\bar{s} \leq 0$, then $\Delta u \geq 0$; hence, $u$ is a positive subharmonic function. In our case, $u>0$ according to the definition given above; thus, $(M, g)$ has a finite volume. Thus, we can formulate the following.

Theorem 6. Let $(M, g)$ be a complete non-compact Riemannian manifold of dimension $n \geq 3$ with non-negative Ricci curvature, and let $(\bar{M}, \bar{g})$ be another Riemannian manifold with a conformally related metric $\bar{g}=u^{4 / n-2} g$ for some smooth function $u>0$ and a diffeomorphism $f: M \rightarrow \bar{M}$. If $u \in L^{p}(M, g)$ for some $p \in(0, \infty)$, then the scalar curvature $\bar{s}$ of $(\bar{M}, \bar{g})$ cannot be non-positive.

Now, let $(M, g)$ be a complete parabolic manifold of dimension $n \geq 3$. In turn, in [23], it was proved that a complete manifold $(M, g)$ is parabolic if and only if any subharmonic function $\phi \in C^{2}(M)$ with finite Dirichlet integral $\int_{M}\|\operatorname{grad} \phi\|^{2} d \mathrm{vol}_{g}$ is constant. Therefore, if we assume in (5) that $\phi=-\sigma, s \geq 0$, and $\bar{s} \leq 0$, then based on the above statement, we conclude that $\phi$ is a subharmonic function, and therefore it is constant. We can formulate the following statement.

Theorem 7. Let $(M, g)$ be a complete parabolic Riemannian manifold of dimension $n \geq 3$ with scalar curvature $s \geq 0$, and let $(\bar{M}, \bar{g})$ be another Riemannian manifold with scalar curvature $\bar{s} \leq 0$. If there exists a conformal diffeomorphism $f:(M, g) \rightarrow(\bar{M}, \bar{g})$ such that $f^{*} \bar{g}=e^{2 \sigma} g$, and $\sigma$ has a finite Dirichlet integral, then $f$ is a homothetic mapping; moreover, $(M, g)$ and $(\bar{M}, \bar{g})$ have zero scalar curvature.

This theorem complements Theorem 3 on conformal mappings of parabolic manifolds. 
Recall that a complete Riemannian manifold of finite volume is an example of a parabolic manifold. On the other hand, in [11] (p. 318), the following was proved: on a complete manifold of finite volume, any subharmonic function with a finite Dirichlet integral is constant. Using this statement, we can formulate the following.

Corollary 3. Let $(M, g)$ be an $n$-dimensional $n \geq 3$ complete Riemannian manifold with finite volume and scalar curvature $s \geq 0$, and let $(\bar{M}, \bar{g})$ be another Riemannian manifold with scalar curvature $\bar{s} \leq 0$. Suppose that there exists a conformal diffeomorphism $f:(M, g) \rightarrow(\bar{M}, \bar{g})$ defined by $f^{*} \bar{g}=e^{2 \sigma} g$. If $\sigma$ has a finite Dirichlet integral, then $f$ is a homothetic mapping; moreover, $(M, g)$ and $(\bar{M}, \bar{g})$ have zero scalar curvature.

Remark 5. The use of the Dirichlet integral in the study of conformal mappings is new, which guarantees us the originality of the results obtained.

\section{An Application of the Theory of Convex Functions to the Study of Conformal Mappings}

Here, we apply two important theorems of the theory of convex functions on complete Riemannian manifolds (see [21,24]) to the study of conformal mappings. Recall that $u \in C^{2}(M)$ is a convex function if its Hessian

$$
\operatorname{Hess}_{g} u:=\nabla d u
$$

is positive semi-definite. Convex functions are an example of subharmonic functions. Using the above definition and the theory of convex functions, we can formulate the following theorem and its corollary.

Theorem 8. Let $f:(M, g) \rightarrow(\bar{M}, \bar{g})$ be a non-homothetic conformal diffeomorphism of complete Riemannian manifold $(M, g)$ onto $(\bar{M}, \bar{g})$ such that $f^{*} \bar{S} \geq S$ for the Schouten tensors $S$ and $\bar{S}$ of $(M, g)$ and $(\bar{M}, \bar{g})$, respectively. Then $(M, g)$ has infinite volume.

Proof. By conditions and (1), Equation (4) can be rewritten in the following form:

$$
\operatorname{Hess}_{g} \sigma=S-\bar{S}+d \sigma \otimes d \sigma-1 / 2\|\operatorname{grad} \sigma\|^{2} g .
$$

Putting $\sigma=-\ln u$ for the associated function $u>0$, we obtain $\bar{g}=u^{-2} g$. Therefore, Equation (3) can be rewritten in the following form:

$$
\operatorname{Hess}_{g} u=u \cdot(\bar{S}-S)+(u / 2)\|\operatorname{grad} \sigma\|^{2} g .
$$

From (7), we conclude that if $\bar{S} \geq S$ (that is $\bar{S}-S$ is a non-negative definite symmetric tensor), then Hess $g u \geq 0$. Hence, $u$ is a convex function. On the other hand, Yau's theorem [21] states that a complete Riemannian manifold admitting a non-constant convex function has infinite volume.

Recall the following theorem of Bishop and O'Neill [24]: if $(M, g)$ is a connected complete Riemannian manifold of finite volume, then any convex function on $(M, g)$ is constant. Therefore, using Theorem 8 , we obtain the following corollary.

Corollary 4. Let $f:(M, g) \rightarrow(\bar{M}, \bar{g})$ be a conformal diffeomorphism of a complete Riemannian manifold $(M, g)$ of finite volume onto another Riemannian manifold $(\bar{M}, \bar{g})$. If $f^{*} \bar{S} \geq S$ for the Schouten tensors $S$ and $\bar{S}$ of $(M, g)$ and $(\bar{M}, \bar{g})$, respectively, then $f$ is a homothetic mapping.

Remark 6. From the inequality $\bar{S} \geq S$, we obtain $e^{2 \sigma} \bar{s} \geq s$. Therefore, if $\bar{s} \geq 0$ and $s \leq 0$, then the inequality $e^{2 \sigma} \bar{s} \geq s$ holds. This fact agrees with the conditions of Theorem 3 and Proposition 1. 


\section{An Application to the Study of Conformal Transformations of the Mixed Scalar Curvature}

There are three kinds of sectional curvatures for a pseudo-Riemannian manifold $(M, g)$ endowed with a smooth distribution (sub-bundle of the tangent bundle): tangential, transversal, and mixed. The mixed plane is spanned by two vectors such that the first (second) vector is tangent (orthogonal) to the distribution. Mixed curvatures stand for the sectional curvatures of mixed planes. This concept has a long history and many applications, e.g., [25].

Let $\mathcal{D}$ and $\overline{\mathcal{D}}$ be $p$-dimensional distributions on connected $n$-dimensional Riemannian manifolds $(M, g)$ and $(\bar{M}, \bar{g})$ with the Levi-Civita connections $\nabla$ and $\bar{\nabla}$ and the curvature tensors $R$ and $\bar{R}$, respectively. Let $f: M \rightarrow \bar{M}$ be a diffeomorphism of $M$ onto $\bar{M}$ preserving the distributions, i.e., for any point $x \in M$, the image $f_{*}\left(\mathcal{D}_{x}\right)$ is $\overline{\mathcal{D}}_{f(x)}$. Below, we will assume that $f$ is a conformal diffeomorphism; thus, $\bar{g}=\left(1 / \phi^{2}\right) g$, i.e., $\phi=e^{-\sigma}$ (see (1)), for $f$-adjusted common coordinates on $M$ and $\bar{M}$. Let $\mathcal{D}^{\perp}$ be the orthogonal complement of $\mathcal{D}$ in $T M$. Let $\left(e_{1}, \ldots, e_{n}\right)$ be an adapted local orthonormal frame, i.e., $e_{i} \in \mathcal{D}$ for $i=1, \ldots, p$, and $e_{a} \in \mathcal{D}^{\perp}$ for $a=p+1, \ldots, n$.

The mixed scalar curvature of a distribution $\mathcal{D}$ on a Riemannian manifold $(M, g)$ is an averaged mixed sectional curvature, i.e., the following function on $M$ :

$$
s_{\text {mix }}=\sum_{a, i} g\left(R\left(e_{a}, e_{i}\right) e_{a}, e_{i}\right) .
$$

To avoid some technical difficulties, assume below that $n=2 p$; thus, $\mathcal{D}$ and its orthogonal complement $\mathcal{D}^{\perp}$ become $p$-dimensional distributions corresponding to an almost paracomplex structure on $(M, g)$, and similarly for $\overline{\mathcal{D}}$ and $\overline{\mathcal{D}}^{\perp}$ on $(\bar{M}, \bar{g})$, see Remark 7 .

Remark 7. An almost paracomplex structure on a manifold $M$ of dimension $n=2 p$ is a continuous field of automorphisms of tangent spaces, the square of which is the identity operator, and the eigensubspaces have dimension $p$ (see [26-28]). This structure is a special case of an almost product structure and is the antipode of an almost complex structure. Therefore, new facts for the geometry of paracomplex manifolds will follow from the statements proved below.

The mixed scalar curvatures of $(M, g, \mathcal{D})$ and $(\bar{M}, \bar{g}, \overline{\mathcal{D}})$ under conformal diffeomorphism preserving the distribution are related by the following formula (see [29]):

$$
p \phi \Delta \phi=-\phi^{2} s_{\text {mix }}+\bar{s}_{\text {mix }}+p^{2}\|\nabla \phi\|^{2} .
$$

Recall the following theorem (see [22]): "let $u>0$ be a smooth function on a complete Riemannian manifold $(M, g)$ such that $(q-1) u \Delta u \geq 0$, where $q$ is a positive constant number, then for $q \neq 1$ we have either $\int_{M} u^{q} d \operatorname{vol}_{g}=\infty$, or $u$ is a constant". Applying this theorem to the above formula, we obtain a Liouville-type theorem.

Theorem 9. Let $\mathcal{D}$ be a $p$-dimensional $(p>1)$ distribution on a $2 p$-dimensional complete Riemannian manifold $(M, g)$. Then, there are no conformal (non-homothetic) transformations of the metric $\bar{g}=\left(1 / \phi^{2}\right) g$ with a positive smooth function $\phi \in L^{q}(M, g)$ for $q \neq 1$ such that $\bar{s}_{\text {mix }} \geq \phi^{2} s_{\text {mix }}$ and, in particular, when $\bar{s}_{\text {mix }} \geq 0$ and $s_{\text {mix }} \leq 0$.

Changing variables $1 / \phi^{2}=u^{4 /(n-2)}$, where $u>0$ is a function on $M$, we rewrite (8) in the following form (see [29] (Corollary 1)):

$$
\Delta u=\frac{n-2}{n}\left(u s_{\text {mix }}-u^{\frac{n+2}{n-2}} \bar{s}_{\text {mix }}\right) .
$$

Therefore, if $s_{\text {mix }} \geq 0$ and $\bar{s}_{\text {mix }} \leq 0$, then $u$ is a positive superharmonic function. Therefore, we can formulate the following theorem. 
Theorem 10. Let $\mathcal{D}$ be a $p$-dimensional $(p>1)$ distribution on a $2 p$-dimensional complete Riemannian manifold $(M, g)$ such that $s_{\text {mix }} \leq 0$. Then, there are no conformal (non-homothetic) transformations of the metric $g$ such that $\bar{s}_{\text {mix }} \geq 0$.

In particular, if $M$ is a compact manifold, then the following two statements hold.

Corollary 5. Let $\mathcal{D}$ be a $p$-dimensional $(p>1)$ distribution on a $2 p$-dimensional compact Riemannian manifold $(M, g)$ such that $s_{\text {mix }} \leq 0$ (resp., $\left.s_{\text {mix }} \geq 0\right)$. Then, there are no conformal (non-homothetic) transformations of $g$ such that $\bar{s}_{\operatorname{mix}} \geq 0\left(\right.$ resp., $\left.\bar{s}_{\operatorname{mix}} \leq 0\right)$.

Corollary 6. Let $g$ and $\bar{g}$ be two conformally equivalent metrics on a $2 p$-dimensional compact manifold $M$ with a $p$-dimensional $(p>1)$ distribution. If both metrics have non-vanishing mixed scalar curvatures, i.e., $s_{\text {mix }} \neq 0$ and $\bar{s}_{\text {mix }} \neq 0$ everywhere, then these curvatures have the same sign.

\section{Conclusions}

In conclusion, we add that the classical Bochner methods have been significantly developed and successfully applied to Finsler manifolds (see, for example, survey [30]) and Lorentzian manifolds, including the theory of relativity (see, for example, [31-33]) over the past 40 years. However, we have already entered the era of geometric analysis and its applications, quite recently, to the use of nonlinear partial differential equations to study geometric and topological properties of submanifolds of Euclidean space and complete Riemannian manifolds. In the 1980s, fundamental contributions to this theory were made by K. Uhlenbeck, C. Taubes, S.-T. Yau, R. Schoen, and R. Hamilton, initiating a particularly productive era of geometric analysis that continues to this day, e.g., [34]. A well-known achievement was the solution of the H. Poincaré conjecture by G. Perel'man, completing the program started and carried out by R. Hamilton (see [35]). Geometric analysis awaits new applications.

Author Contributions: Methodology, V.R., I.T. and S.S.; investigation, writing-review and editing. All authors have read and agreed to the published version of the manuscript.

Funding: This research received no external funding.

Conflicts of Interest: The authors declare no conflict of interest.

\section{References}

1. Yano, K.; Bochner, S. Curvature and Betti Numbers; Princeton University Press: Princeton, NJ, USA, 1953.

2. Besse, A.L. Einstein Manifolds; Springer: Berlin, Germany, 1987.

3. Gallot, S.; Meyer, D. Opérateur de courbure et laplacien des formes différentielles d'une variété riemannianne. J. Math. Pures. Appl. 1975, 54, 259-284.

4. Wu, H.-H. The Bochner Technique in Differential Geometry, Classical Topics in Mathematics; Higher Education Press: Beijing, China, 2018; Volume 6.

5. Petersen, P. Riemannian Geometry, 3rd ed.; Springer AG: New York, NY, USA, 2016.

6. Yano, K. Integral Formulas in Riemannian Geometry; Marcel Dekker: New York, NY, USA, 1970.

7. Berard, P. H. From vanishing theorems to estimating theorems: The Bochner technique revisited. Bull. AMS 1988, 19, 371-406. [CrossRef]

8. Pigola, S.; Rigoli, M.; Setti, A.G. Vanishing and Finiteness Results in Geometric Analysis: A Generalization of the Bochner Technique; Progress in Mathematics; Birkhäuser Verlag AG: Basel, Switzerland, 2008; Volume 266.

9. Pigola, S.; Rigoli, M.; Setti, A.G. Maximum Principles on Riemannian Manifolds and Applications; AMS: Providence, RI, USA, 2005.

10. Grigor'yan, A. Heat Kernel and Analysis on Manifolds; AMS/IP: Boston, FL, USA, 2009.

11. Schoen, R.; Yau, S.T. Lectures on Harmonic Maps; International Press: Boston, FL, USA, 1994.

12. Mikeš, J.; Rovenski, V.; Stepanov, S.E. An example Lichnerowicz-type Laplacian. Ann. Glob. Anal. Geom. 2020, 58, 19-34. [CrossRef]

13. Stepanov, S.E.; Mikeš, J. Liouville-type theorems for some classes of Riemannian almost product manifolds and for special mappings of Riemannian manifolds. Differ. Geom. Appl. 2017, 54, 111-121. [CrossRef]

14. Narasimhan, R. Analysis on real and complex manifolds. In Advanced Studies in Pure Mathematics; Masson \& Cie, Éditeurs: Paris, France; North-Holland Publishing Co.: Amsterdam, The Netherlands, 1968; Volume 1. 
15. Yau, S.-T. Remarks on conformal transformations. J. Diff. Geom. 1973, 8, 369-381. [CrossRef]

16. Eisenhart, L.P. Riemannian Geometry; Princeton University Press: Princeton, RI, USA, 1949.

17. Grigor'yan, A. Analytic and geometric background of recurrence and non-explosion of the Brownian motion on Riemannian manifolds. Bull. Amer. Math. Soc. 1999, 36, 135-249. [CrossRef]

18. Adams, S.R. Superharmonic functions on foliations. Trans. AMS 1992, 330, 625-635. [CrossRef]

19. Ni, L. Vanishing theorems on complete Kahler manifolds and their applications. J. Differ. Geom. 1998, 50, 89-122. [CrossRef]

20. Yau, S.-T. On the heat kernel of a complete Riemannian manifold. J. Math. Pures Appl. 1978, 57, 191-201.

21. Yau, S.T. Non-existence of continuous convex functions on certain Riemannian manifolds. Math. Ann. 1974, 207, 269-270. [CrossRef]

22. Yau, S.T. Some function-theoretic properties of complete Riemannian manifolds and their applications to geometry. Indiana Univ. Math. J. 1976, 25, 659-670; Erratum in 1982, 31, 607. [CrossRef]

23. Pigola, S.; Rigoli, M.; Setti, A.G. Aspect of potential theory on manifolds, linear and non-linear. Milan J. Math. 2008, 76, 229-256. [CrossRef]

24. Bishop, R.L.; O'Neill, B. Manifolds of negative curvature. Trans. Am. Math. Soc. 1969, 145, 1-9. [CrossRef]

25. Rovenski, V. Foliations on Riemannian Manifolds and Submanifolds; Birkhäuser Boston: Boston, MA, USA, 1998.

26. Cruceanu, V.; Fortuny, P.; Gadea, P.M. A survey on paracomplex geometry. Rocky Mountain J. Math. 1996, 26, 83-115. [CrossRef]

27. Kornev, E.S. The bundle of paracomplex structures. Sib. Math. J. 2020, 61, 687-696. [CrossRef]

28. Alekseevskiŭ, D.V.; Medori, K.; Tomassini, A. Homogeneous para-Kählerian Einstein manifolds. Russian Math. Surv. 2009, 64, 1-43. [CrossRef]

29. Rovenski, V. On solutions to equations with partial Ricci curvature. J. Geom. Phys. 2014, 86, 370-382. [CrossRef]

30. Xiao, J.; Qiu, C.; Zhong, T. Bochner-Kodaira techniques on Kähler Finsler manifolds. Chin. Ann. Math. Ser. B 2015, 36, 125-140. [CrossRef]

31. Romero, A. The introduction of Bochner's technique on Lorentzian manifolds. Nonlinear Anal. 2001, 47, 3047-3059. [CrossRef]

32. Stepanov, S.E. Vanishing theorems in affine, Riemann and Lorentzian geometries. J. Math. Sci. (N. Y.) 2007, 141, 929-964. [CrossRef]

33. Petersen, P.; Wink, M. New curvature conditions for the Bochner technique. Invent. Math. 2021, 224, 33-54. [CrossRef]

34. Schoen, R.; Yau, S.-T, Lectures on Differential Geometry; International Press of Boston: Boston, FL, USA, 2010.

35. Topping, P. Lectures on the Ricci Flow; Cambridge University Press: Cambridge, MA, USA, 2006. 